\title{
Effect of Lactate and Lactate Clearance Condition on the Prognosis of Sepsis in Children
}

\section{Running title: Lactate and Lactate Clearance in Sepsis in Children}

\author{
D M Kamrul Hasan ${ }^{1}$, Shafayat Hossain Evan ${ }^{1}$, Md Rezaul Karim², Xin Yi Men ${ }^{1}$, Zhang \\ Huifeng ${ }^{1}$, Chen Yuan ${ }^{1 *}$
}
${ }^{1}$ Department of Pediatrics, Second Affiliated Hospital of Hebei Medical University, Shijiazhuang, Hebei, China
${ }^{2}$ Hubei Key Laboratory of Embryonic Stem Cell Research, Institute of Neuroscience, Hubei University of Medicine, Shiyan, Hubei, China
*Correspondence to: Prof. Dr. Chen Yuan, Ph.D.; Department of Pediatrics, Second Affiliated Hospital of Hebei Medical University, Shijiazhuang, Hebei, China; Email: pediatric@126.com

\begin{abstract}
Objectives: To investigate the value of early lactate dynamic monitoring index in predicting prognosis of patients with sepsis and septic shock. Methods: We performed our test on 50 patients. Out of 50 patients, 28 are male, and 22 are female. Prospectively studied pediatric patients with septic shock were performed. Vital signs, Lactate clearance, were obtained at presentation $6 \mathrm{~h}, 12 \mathrm{~h}, 24 \mathrm{~h}$ over the first $48 \mathrm{~h}$ of hospitalization. The therapy received, outcome parameters of mortality and duration of hospitalization were recorded. Results: The statistical data and comparative analysis showed that an average of 16.88 days after admission, 5 patients have died, 17 patients are poorly prognosis leaves the hospital, and the remaining 28 are recovered and discharged. The primary outcome variable of mean 16 days hospitalization mortality rate was $10 \%$. Poor prognosis $34 \%$ and fully recovery $56 \%$ were observed. In this retrospective cohort study, a lactate level of more than $2.5 \mathrm{mmol} / \mathrm{L}$ was the best threshold to predict 28-day mortality among severe sepsis and septic shock patients. In our research, we found mean LC $6 \mathrm{~h} 3.08 \mathrm{mmol} / \mathrm{L}$, and after $48 \mathrm{~h}$ mean it is $1.79 \mathrm{mmol} / \mathrm{L}$. Significant LC $6 \mathrm{~h}$ found, which is $8.08 \mathrm{mmol} / \mathrm{L}$ in the death group patient where $48 \mathrm{~h} \mathrm{mmol} / \mathrm{L}$ shows significant high. Poor prognosis also presents a clinical increase of lactate level high in the LC $6 \mathrm{~h}$ analysis, which is $3.32 \mathrm{mmol} / \mathrm{L}$. Recovered patients showed a significant improvement after administering treatment depending on the patient organ involvement and good decrease of lactate reports achieved, which is $1.20 \mathrm{mmol} / \mathrm{L}$, where admission reports show it was
\end{abstract}


$1.91 \mathrm{mmol} / \mathrm{L}$ in LC $6 \mathrm{~h}$. Mean Heart rate $94 / 51 \mathrm{mmhg}$, pulse 119 , temperature $39^{\circ} \mathrm{C}$, respiratory rate 32.26, and urine output $456 \mathrm{ml}$ recorded during our study. Death patient shows a remarkable detonation of those reports but has a significant clinical report with the recovered patients. Conclusion: The early lactate dynamic monitoring index has a high value in predicting sepsis and septic shock patients' prognosis, thus worth popularizing.

Keywords: Sepsis, Septic shock, Lactic acid, Dynamic monitoring, Prognosis

\section{INTRODUCTION}

Sepsis is defined as a life-threatening organ dysfunction occurred by a deregulated host response to infection ${ }^{1}$. The third international consensus defined septic shock as a subset of sepsis in which particularly profound circulatory, cellular, and metabolic abnormalities are associated with an increased risk of mortality than sepsis alone ${ }^{1}$. Early recognition of patients at risk and aggressive treatment within the first few $\mathrm{h}$ after presentation may prevent the invariable progression and poor outcome, manifested clinically by end-organ damage, failure of multiple organ systems, and death ${ }^{2-3}$. Elevated serum lactate levels reflect the anaerobic metabolism related to cellular hypoxia and are thought to be an important marker of impaired tissue perfusion in patients with septic shock ${ }^{4}$. Small observational studies in adults and children have demonstrated that lactate can correlate with the severity of shock and prognosis in sepsis ${ }^{5-6}$. In contrast, the sensitivity and specificity of single lactate concentrations as markers of tissue hypoperfusion have been debated ${ }^{7-8}$. Studies have shown that serial measurements or lactate clearance (LC) over time may be better prognosticators of organ failure and mortality ${ }^{9-13}$.

Further, studies in adults have established the use of lactate and LC as a diagnostic, prognostic, and therapeutic marker of global tissue hypoxia in sepsis and septic shock, however literature regarding its possible prognosticator role in pediatric septic shock isscanty ${ }^{13}$. Further, there is no data regarding the comparison of $\mathrm{LC}$ at different intervals during the resuscitation of pediatric septic shock. This study was designed to examine the clinical utility of LC as an indicator of mortality in pediatric septic shock, and to compare the efficiency of LC at 6,12, and $24 \mathrm{~h}$ for predicting in-hospital and 60-day mortality. We also defined a cutoff for LC that is associated with improved outcome after 6 and $24 \mathrm{~h}$ of intensive care intervention. Patients were intubated and mechanically ventilated as required. We excluded neonates and patients above 17 years of age in the study. 


\section{MATERIALS AND METHODS}

\subsection{Study design}

This prospective observational study was performed in the pediatric intensive care unit (PICU) of Department of Pediatrics at Hebei Medical University 2nd hospital, one of the provincial, medical hospital centers in Hebei province Shijiazhuang city. The study was conducted over a period of 1.5 years from June 2018 to Dec 2019. The hospital ethical committee approved the study. Informed consent was taken from the parents/guardians of the study patients.

\subsection{Participants}

One hundred and twelve consecutive children in the age group of one month to 17 years, diagnosed with septic shock, constituted the study group. Sepsis and septic shock were defined as per International pediatric sepsis consensus definitions. (1) Included patients were admitted through the emergency department and immediately shifted to PICU. They received central venous and arterial catheterization and were managed as per the prescribed guidelines for goaldirected stepwise management of hemodynamic support in infants and children. (2) Targeted resuscitation end-points were as follows:

1. Blood pressure (systolic pressure at least 5th percentile)

For age: $60 \mathrm{mmHg}<1$ month of age, $70 \mathrm{mmHg}$ ( 2 ages in years) in children 1 month to 10 years of age, $90 \mathrm{mmHg}$ in children (10 years of age or older).

2. Central and peripheral pulses (strong, distal pulse sequel to central pulses).

3. Normal mental status.

4. Adequate skin perfusion (warm, and capillary refill $<2 \mathrm{~s}$ ).

5. Urine output $51 \mathrm{~mL} / \mathrm{kg} / \mathrm{h}$ (after effective circulating volume is restored).

\subsection{Data collection and data elements}

The primary outcome variable was the 60-day mortality. Demographic characteristics and admission diagnoses were recorded at the baseline. Glasgow Coma Scale (GCS), baseline vital signs (temperature, heart rate, mean arterial pressure, central venous pressure), arterial lactate, laboratory values, and therapy received were recorded. The severity of critical illness was assessed using the Pediatric Risk of Mortality III (PRISM III) score within $24 \mathrm{~h}$ of hospital admission. Organ dysfunction was analyzed and followed using pediatric logistic organ dysfunction (PELOD)score at 0 (at presentation), 6, 24, 48, and $72 \mathrm{~h}$ while in the hospital. 
Lactate levels were measured on admission to PICU along with other baseline investigations and septic work-up, and repeat lactate levels were taken at 6,12 , and $24 \mathrm{~h}$ post-admission.

\section{RESULTS}

Table 1: Lactate Range and Clearance

\begin{tabular}{|c|c|c|c|c|c|c|c|}
\hline $\mathbf{S} / \mathbf{N}$ & $\begin{array}{c}\text { Patient } \\
\text { number }\end{array}$ & $\begin{array}{c}6 \mathrm{~h} . \\
\mathrm{mmol} / \mathrm{L}\end{array}$ & $\begin{array}{c}12 \mathrm{~h} \\
\mathrm{mmol} / \mathrm{IL}\end{array}$ & $\begin{array}{c}24 h \\
\text { mmol }\end{array}$ & $\begin{array}{c}48 \mathrm{~h} \\
\mathrm{mmol} / \mathrm{L}\end{array}$ & Prognosis & Outcome \\
\hline 1 & 2219774 & 7.8 & 2.8 & 2.4 & 1.9 & Death & \\
\hline 2 & 2218957 & 15 & 15 & 15 & 15 & Death & \\
\hline 3 & 2106795 & 0.8 & 1.3 & 1.2 & & Death & \\
\hline 4 & 2110787 & 1.8 & 2.2 & 1.4 & 2.6 & Death & \\
\hline 5 & 2056034 & 15 & 1.77 & 1.27 & 1.9 & Death & \\
\hline 6 & 2214460 & 5.2 & & & & Poor & Stop treatment \\
\hline 7 & 2213627 & 3.3 & 2.7 & 1.4 & 0.9 & Poor & \\
\hline 8 & 2202400 & 2.4 & 1.16 & 0.6 & 0.9 & Poor & Stop treatment \\
\hline 9 & 2199578 & 1.2 & 1.04 & 0.7 & 1.1 & Poor & Stop Treatment \\
\hline 10 & 2200766 & 7.41 & 8.4 & 9 & 8.4 & Poor & Stop Treatment \\
\hline 11 & 2173103 & 2.46 & 2.1 & 1.2 & 1.5 & Poor & Stop Treatment \\
\hline 12 & 2160501 & 4.4 & 3.5 & 3.9 & 2.9 & Poor & Stop Treatment \\
\hline 13 & 2165571 & 12.7 & 1.9 & 2.2 & 2.5 & Poor & Stop Treatment \\
\hline 14 & 2115119 & 0.7 & 0.74 & 0.9 & 1.13 & Poor & Stop Treatment \\
\hline 15 & 2094981 & 1.7 & 1.2 & 1.6 & 1.1 & Poor & Stop Treatment \\
\hline 16 & 2135402 & 3.39 & 4.2 & 4.7 & 2.3 & Poor & Stop Treatment \\
\hline 17 & 2124264 & 1.6 & 1.7 & 1.5 & 0.9 & Poor & Stop Treatment \\
\hline 18 & 2105185 & 1.5 & 1.5 & 1.5 & 1.8 & Poor & Stop Treatment \\
\hline 19 & 2086416 & 1.7 & 1.7 & 1.8 & 0.92 & Poor & Stop treatment \\
\hline 20 & 2085364 & 2.2 & 2.81 & 1.64 & 1.33 & Poor & Stop Treatment \\
\hline 21 & 2054021 & 3.01 & 2.43 & 2.92 & 1.22 & Poor & Stop Treatment \\
\hline 22 & 2094981 & 1.7 & 3.1 & 1.6 & 1.8 & Poor & Stop Treatment \\
\hline 23 & 2216470 & 1 & 1.5 & 1.4 & 1.1 & Good & \\
\hline 24 & 2197241 & 1.9 & 0.3 & 0.7 & 0.9 & Good & \\
\hline 25 & 2207437 & 1.1 & 0.9 & 0.8 & 0.6 & Good & \\
\hline 26 & 2192077 & 1.5 & 0.9 & 1.2 & 0.8 & Good & \\
\hline 27 & 2176797 & 1.2 & 1.4 & 0.4 & 0.6 & Good & \\
\hline 28 & 2183460 & 1.0 & 3.2 & 1.3 & 1.2 & Good & \\
\hline 29 & 2178301 & 1.2 & 2.3 & 2.1 & 0.9 & Good & \\
\hline 30 & 2002726 & 1.1 & 2.3 & 1.4 & 1.9 & Good & \\
\hline 31 & 2169128 & 1.0 & 0.6 & 1.1 & 0.8 & Good & \\
\hline 32 & 2157435 & 1.2 & 3.2 & 2.1 & 2.7 & Good & \\
\hline 33 & 2151377 & 9.8 & 3.2 & 1.0 & 2.0 & Good & \\
\hline 34 & 2145015 & 1.4 & 0.9 & 1.2 & 0.6 & Good & \\
\hline 35 & 2131298 & 1.5 & 1.9 & 0.5 & 1.54 & Good & \\
\hline 36 & 2141183 & 1.9 & 1.2 & 1.5 & 1.1 & Good & \\
\hline 37 & 2137040 & 0.71 & 1.2 & 1.7 & 0.98 & Good & \\
\hline 38 & 1638611 & 5.1 & 7.0 & 3.5 & 2.3 & Good & \\
\hline 39 & 2122281 & 2.8 & 2.1 & 1.9 & 2.0 & Good & \\
\hline 40 & 2119718 & 1.1 & 0.9 & 1.0 & 1.2 & Good & \\
\hline 41 & 2122753 & 5.6 & 1.2 & 1.3 & 0.9 & Good & \\
\hline 42 & 2114428 & 1.4 & 1.7 & 1.1 & 0.7 & Good & \\
\hline 43 & 2115119 & 0.7 & 2.1 & 0.6 & 0.9 & Good & \\
\hline 44 & 2102797 & 0.7 & 0.9 & 0.8 & 1.0 & Good & \\
\hline 45 & 2097573 & 1.5 & 1.1 & 1.2 & 1.7 & Good & \\
\hline 46 & 2088984 & 0.8 & 1.2 & 0.7 & 0.3 & Good & \\
\hline 47 & 2095684 & $\begin{array}{l}0.0 \\
1.7\end{array}$ & 1.2 & 1.8 & 1.2 & Good & \\
\hline 48 & 2223159 & 2.0 & 1.0 & $\begin{array}{l}1.0 \\
1.0\end{array}$ & 1.33 & Good & \\
\hline 49 & 2083190 & 2.3 & 2.3 & 0.9 & 1.2 & Good & \\
\hline 50 & 2067318 & 2.0 & 2.83 & 2.1 & 1.4 & Good & \\
\hline
\end{tabular}


Table 2. Age Sex Hospital Arrival and Leaving time.

\begin{tabular}{|c|c|c|c|c|c|c|}
\hline $\mathbf{S} / \mathbf{N}$ & $\begin{array}{l}\text { Patient } \\
\text { number }\end{array}$ & Sex & Age & Arrival time & Leaving time & $\begin{array}{c}\text { Duration of } \\
\text { stay-Day }\end{array}$ \\
\hline 1 & 2219774 & $\mathrm{M}$ & 2.0 & $26 / 11 / 2019$ & $03 / 12 / 2019$ & 07 \\
\hline 2 & 2218957 & M & 0.3 & $24 / 11 / 2019$ & $27 / 11 / 2019$ & 03 \\
\hline 3 & 2106795 & M & 2.0 & $10 / 5 / 2019$ & $12 / 05 / 2019$ & 02 \\
\hline 4 & 2110787 & M & 11 & $25 / 12 / 2018$ & 07/01/2019 & 13 \\
\hline 5 & 2056034 & M & 1.9 & 05/09/2018 & $30 / 11 / 2018$ & 85 \\
\hline 6 & 2214460 & $\mathrm{~F}$ & 13 & 10/11/2019 & $13 / 11 / 2019$ & 03 \\
\hline 7 & 2213627 & M & 3.0 & $07 / 11 / 2019$ & 08/11/2019 & 01 \\
\hline 8 & 2202400 & M & 7.0 & 03/10/2019 & $25 / 10 / 2019$ & 22 \\
\hline 9 & 2199578 & $\mathrm{~F}$ & 0.1 & 22/09/2019 & 20/10/2019 & 28 \\
\hline 10 & 2200766 & $\mathrm{~F}$ & 0.7 & 25/09/2019 & 01/10/2019 & 06 \\
\hline 11 & 2173103 & M & 0.1 & 02/07/2019 & 05/07/2019 & 03 \\
\hline 12 & 2160501 & M & 5.0 & 23/05/2019 & 28/06/2019 & 35 \\
\hline 13 & 2165571 & $\mathrm{~F}$ & 0.3 & 09/06/2019 & 21/06/2019 & 11 \\
\hline 14 & 2115119 & $\mathrm{~F}$ & 12 & 07/01/2019 & $11 / 01 / 2019$ & 04 \\
\hline 15 & 2094981 & $\mathrm{~F}$ & 9.0 & 07/01/2019 & 28/03/2019 & 80 \\
\hline 16 & 2135402 & $\mathrm{~F}$ & 4.0 & $11 / 03 / 2019$ & $18 / 03 / 2019$ & 07 \\
\hline 17 & 2124264 & $\mathrm{~F}$ & 6.0 & $10 / 02 / 2019$ & $16 / 02 / 2019$ & 06 \\
\hline 18 & 2105185 & $\mathrm{~F}$ & 4.0 & $08 / 12 / 2018$ & 07/01/2019 & 30 \\
\hline 19 & 2086416 & M & 0.1 & $13 / 10 / 2018$ & 06/11/2018 & 23 \\
\hline 20 & 2085364 & M & 0.4 & 09/10/2018 & $22 / 10 / 2018$ & 12 \\
\hline 21 & 2054021 & M & 2.0 & 03/07/2018 & $10 / 07 / 2018$ & 07 \\
\hline 22 & 2094981 & $\mathrm{~F}$ & 9.0 & 07/01/2019 & 28/03/2019 & 79 \\
\hline 23 & 2216470 & M & 6.0 & $15 / 11 / 2019$ & $25 / 11 / 2019$ & 10 \\
\hline 24 & 2197241 & $\mathrm{~F}$ & 8.0 & $12 / 11 / 2019$ & $15 / 11 / 2019$ & 03 \\
\hline 25 & 2207437 & $\mathrm{~F}$ & 0.1 & $18 / 10 / 2019$ & $02 / 11 / 2019$ & 15 \\
\hline 26 & 2192077 & $\mathrm{~F}$ & 13 & 28/08/2019 & 03/09/2019 & 06 \\
\hline 27 & 2176797 & $\mathrm{~F}$ & 06 & 13/07/2019 & 19/08/2019 & 06 \\
\hline 28 & 2183460 & $\mathrm{~F}$ & 10 & 02/08/2019 & 09/08/2019 & 06 \\
\hline 29 & 2178301 & M & 0.8 & 17/07/2019 & 22/07/2019 & 05 \\
\hline 30 & 2002726 & M & 4.0 & 25/06/2019 & 07/07/2019 & 13 \\
\hline 31 & 2169128 & M & 4.0 & 19/06/2019 & 26/06/2019 & 07 \\
\hline 32 & 2157435 & M & 7.0 & $13 / 05 / 2019$ & 22/05/2019 & 09 \\
\hline 33 & 2151377 & M & 6.0 & 25/04/2019 & $13 / 05 / 2019$ & 20 \\
\hline 34 & 2145015 & M & 5.0 & 08/04/2019 & 01/05/2019 & 21 \\
\hline 35 & 2131298 & $\mathrm{~F}$ & 4.0 & $31 / 03 / 2019$ & 25/04/2019 & 25 \\
\hline 36 & 2141183 & M & 1.0 & 01/04/2019 & 18/04/2019 & 17 \\
\hline 37 & 2137040 & M & 1.0 & $16 / 03 / 2019$ & $26 / 03 / 2019$ & 09 \\
\hline 38 & 1638611 & M & 2.0 & $11 / 02 / 2019$ & $11 / 03 / 2019$ & 30 \\
\hline 39 & 2122281 & M & 3.0 & $30 / 01 / 2019$ & $01 / 03 / 2019$ & 30 \\
\hline 40 & 2119718 & M & 10 & $21 / 01 / 2019$ & $20 / 02 / 2019$ & 31 \\
\hline 41 & 2122753 & $\mathrm{~F}$ & 0.1 & $31 / 01 / 2019$ & $17 / 02 / 2019$ & 17 \\
\hline 42 & 2114428 & $\mathrm{~F}$ & 9.0 & 06/01/2019 & $16 / 01 / 2019$ & 10 \\
\hline 43 & 2115119 & $\mathrm{~F}$ & 12 & 07/01/2019 & $11 / 01 / 2019$ & 04 \\
\hline 44 & 2102797 & M & 1.0 & $01 / 12 / 2018$ & $16 / 12 / 2018$ & 15 \\
\hline 45 & 2097573 & M & 0.9 & $15 / 11 / 2018$ & $27 / 11 / 2018$ & 12 \\
\hline 46 & 2088984 & $\mathrm{~F}$ & 5.0 & $20 / 10 / 2018$ & $09 / 11 / 2018$ & 20 \\
\hline 47 & 2095684 & $\mathrm{~F}$ & 1.0 & $09 / 11 / 2018$ & $18 / 11 / 2018$ & 09 \\
\hline 48 & 2223159 & M & 1.0 & $08 / 12 / 2019$ & $16 / 12 / 2019$ & 08 \\
\hline 49 & 2083190 & $\mathrm{~F}$ & 13 & $04 / 10 / 2018$ & $11 / 10 / 2018$ & 07 \\
\hline 50 & 2067318 & M & 0.2 & $13 / 08 / 2018$ & $24 / 08 / 2018$ & 11 \\
\hline
\end{tabular}


Table 3: Average Blood Pressure, Heart Rate, Temperature, Respiratory Rate \& Urine Output first 24 H

\begin{tabular}{|c|c|c|c|c|c|c|c|}
\hline $\mathbf{S} / \mathbf{N}$ & $\begin{array}{l}\text { Patient } \\
\text { number }\end{array}$ & Sex & $\begin{array}{c}\text { Blood pressure } \\
\text { mm HG }\end{array}$ & $\begin{array}{c}\text { Heart } \\
\text { Rate/Min }\end{array}$ & $\begin{array}{l}\text { Temperatu } \\
\text { re(C) }\end{array}$ & $\begin{array}{c}\text { Respirato } \\
\text { ry rate }\end{array}$ & $\begin{array}{c}\text { Urine } \\
\text { Output }\end{array}$ \\
\hline 1 & 2219774 & $\mathrm{M}$ & $90 / 50$ & 99 & 38.5 & 25 & $210 \mathrm{ml}$ \\
\hline 2 & 2218957 & M & $75 / 40$ & 120 & 39.4 & 35 & $150 \mathrm{ml}$ \\
\hline 3 & 2106795 & M & $95 / 55$ & 102 & 38.3 & 26 & $207 \mathrm{ml}$ \\
\hline 4 & 2110787 & M & $105 / 65$ & 110 & 39.5 & 23 & $750 \mathrm{ml}$ \\
\hline 5 & 2056034 & M & $90 / 55$ & 115 & 39.7 & 27 & $300 \mathrm{ml}$ \\
\hline 6 & 2214460 & $\mathrm{~F}$ & $115 / 70$ & 98 & 39.5 & 20 & $960 \mathrm{ml}$ \\
\hline 7 & 2213627 & M & $92 / 52$ & 110 & 38.6 & 22 & $540 \mathrm{ml}$ \\
\hline 8 & 2202400 & M & $105 / 67$ & 99 & 38.30 & 23 & $650 \mathrm{ml}$ \\
\hline 9 & 2199578 & $\mathrm{~F}$ & $75 / 40$ & 135 & 39.20 & 43 & $175 \mathrm{ml}$ \\
\hline 10 & 2200766 & $\mathrm{~F}$ & $72 / 43$ & 145 & 38.70 & 42 & $290 \mathrm{ml}$ \\
\hline 11 & 2173103 & M & $75 / 45$ & 175 & 39.00 & 45 & $170 \mathrm{ml}$ \\
\hline 12 & 2160501 & M & $110 / 55$ & 110 & 40.01 & 25 & $650 \mathrm{ml}$ \\
\hline 13 & 2165571 & $\mathrm{~F}$ & $85 / 45$ & 138 & 39.70 & 43 & $165 \mathrm{ml}$ \\
\hline 14 & 2115119 & $\mathrm{~F}$ & $100 / 60$ & 102 & 40.10 & 22 & $655 \mathrm{ml}$ \\
\hline 15 & 2094981 & $\mathrm{~F}$ & $105 / 62$ & 95 & 39.50 & 27 & $620 \mathrm{ml}$ \\
\hline 16 & 2135402 & $\mathrm{~F}$ & $104 / 57$ & 111 & 37.90 & 29 & $450 \mathrm{ml}$ \\
\hline 17 & 2124264 & $\mathrm{~F}$ & $104 / 56$ & 125 & 40.20 & 27 & $560 \mathrm{ml}$ \\
\hline 18 & 2105185 & $\mathrm{~F}$ & $79 / 42$ & 125 & 39.70 & 30 & $565 \mathrm{ml}$ \\
\hline 19 & 2086416 & M & $85 / 34$ & 185 & 38.90 & 54 & $110 \mathrm{ml}$ \\
\hline 20 & 2085364 & M & $89 / 34$ & 178 & 39.50 & 56 & $135 \mathrm{ml}$ \\
\hline 21 & 2054021 & M & $97 / 49$ & 135 & 40.00 & 39 & $330 \mathrm{ml}$ \\
\hline 22 & 2094981 & $\mathrm{~F}$ & $95 / 54$ & 103 & 40.01 & 27 & $655 \mathrm{ml}$ \\
\hline 23 & 2216470 & M & $97 / 55$ & 115 & 38.90 & 29 & $570 \mathrm{ml}$ \\
\hline 24 & 2197241 & $\mathrm{~F}$ & $103 / 63$ & 110 & 38.60 & 28 & $585 \mathrm{ml}$ \\
\hline 25 & 2207437 & $\mathrm{~F}$ & $80 / 39$ & 187 & 38.30 & 47 & $120 \mathrm{ml}$ \\
\hline 26 & 2192077 & $\mathrm{~F}$ & $95 / 55$ & 75 & 37.03 & 22 & $710 \mathrm{ml}$ \\
\hline 27 & 2176797 & $\mathrm{~F}$ & $93 / 53$ & 77 & 38.02 & 29 & $645 \mathrm{ml}$ \\
\hline 28 & 2183460 & $\mathrm{~F}$ & $100 / 60$ & 73 & 39.05 & 25 & $660 \mathrm{ml}$ \\
\hline 29 & 2178301 & M & $75 / 39$ & 139 & 39.01 & 47 & $320 \mathrm{ml}$ \\
\hline 30 & 2002726 & M & $90 / 47$ & 115 & 38.80 & 27 & $480 \mathrm{ml}$ \\
\hline 31 & 2169128 & M & $91 / 48$ & 112 & 38.90 & 28 & $470 \mathrm{ml}$ \\
\hline 32 & 2157435 & M & $99 / 63$ & 109 & 39.04 & 27 & $575 \mathrm{ml}$ \\
\hline 33 & 2151377 & M & $102 / 65$ & 111 & 39.03 & 28 & $565 \mathrm{ml}$ \\
\hline 34 & 2145015 & $\mathrm{M}$ & $103 / 63$ & 113 & 38.70 & 26 & $550 \mathrm{ml}$ \\
\hline 35 & 2131298 & $\mathrm{~F}$ & $105 / 67$ & 109 & 38.80 & 27 & $555 \mathrm{ml}$ \\
\hline 36 & 2141183 & M & $98 / 44$ & 125 & 39.30 & 33 & $288 \mathrm{ml}$ \\
\hline 37 & 2137040 & M & $97 / 45$ & 124 & 39.40 & 36 & $290 \mathrm{ml}$ \\
\hline 38 & 1638611 & M & $98 / 43$ & 135 & 38.70 & 36 & $390 \mathrm{ml}$ \\
\hline 39 & 2122281 & M & $100 / 45$ & 133 & 39.10 & 34 & $420 \mathrm{ml}$ \\
\hline 40 & 2119718 & $\mathrm{M}$ & $102 / 62$ & 108 & 37.90 & 25 & $550 \mathrm{ml}$ \\
\hline 41 & 2122753 & $\mathrm{~F}$ & $90 / 39$ & 167 & 38.40 & 50 & $195 \mathrm{ml}$ \\
\hline 42 & 2114428 & $\mathrm{~F}$ & $97 / 55$ & 85 & 39.00 & 28 & $860 \mathrm{ml}$ \\
\hline 43 & 2115119 & $\mathrm{~F}$ & $100 / 67$ & 87 & 39.30 & 27 & $870 \mathrm{ml}$ \\
\hline 44 & 2102797 & M & $95 / 44$ & 119 & 38.40 & 35 & $330 \mathrm{ml}$ \\
\hline 45 & 2097573 & M & $94 / 43$ & 125 & 39.90 & 37 & $320 \mathrm{ml}$ \\
\hline 46 & 2088984 & $\mathrm{~F}$ & $90 / 43$ & 115 & 38.90 & 27 & $590 \mathrm{ml}$ \\
\hline 47 & 2095684 & $\mathrm{~F}$ & $93 / 43$ & 118 & 39.80 & 36 & $335 \mathrm{ml}$ \\
\hline 48 & 2223159 & M & $90 / 45$ & 117 & 40.00 & 39 & $330 \mathrm{ml}$ \\
\hline 49 & 2083190 & $\mathrm{~F}$ & $110 / 68$ & 90 & 39.40 & 18 & $750 \mathrm{ml}$ \\
\hline 50 & 2067318 & $\mathrm{M}$ & $80 / 39$ & 177 & 39.30 & 52 & $190 \mathrm{ml}$ \\
\hline
\end{tabular}




\section{Table 4: Vitals and Urine Output}

\begin{tabular}{lllllll}
\hline S/N & Patient & BP & PULSE & TEMP. (C) & RESPIRATORY & URINE OUTPUT (ML) \\
\hline 1 & All & $94 / 51$ & 119 & 39.06 & 32.26 & 456 \\
2 & Death & $91 / 48$ & 109 & 39.08 & 27.20 & 323 \\
3 & Poor & $93 / 50$ & 127 & 39.03 & 33.76 & 451 \\
4 & Good & $95 / 55$ & 116 & 38.89 & 32.25 & 482 \\
\hline
\end{tabular}

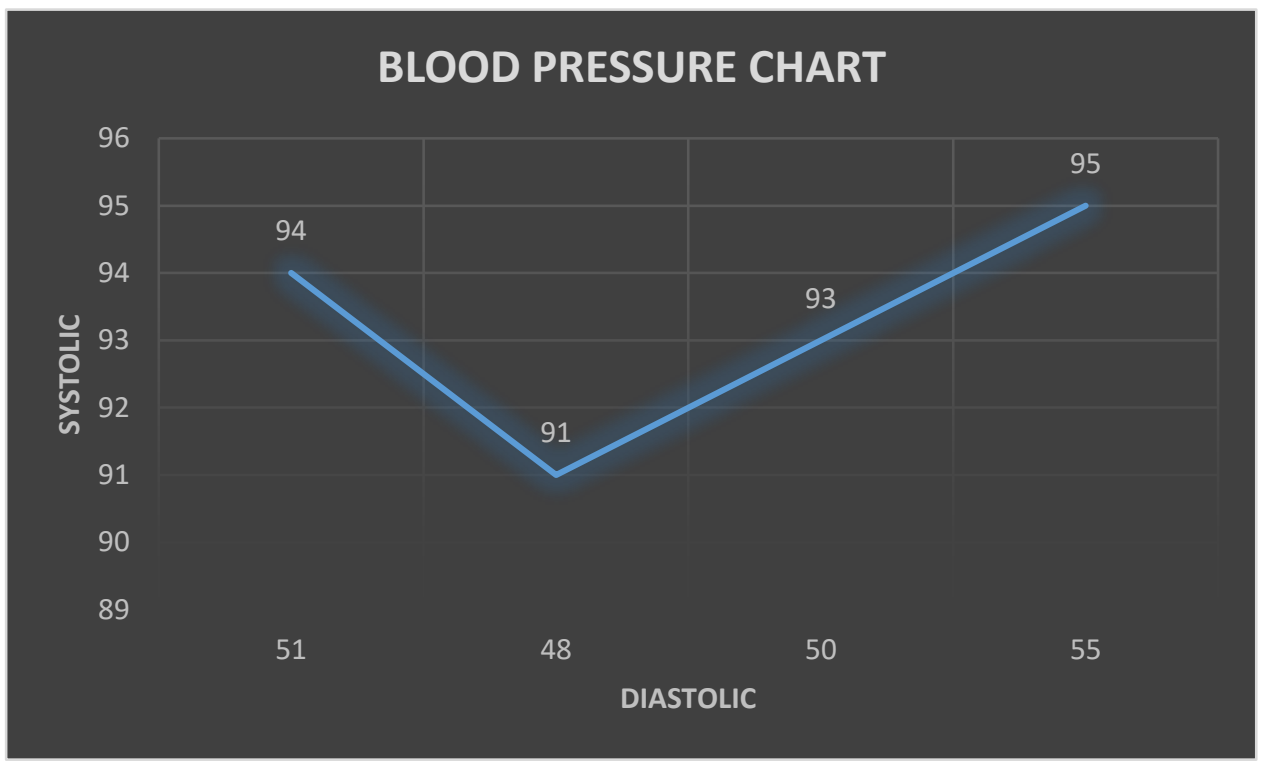

Figure 1: Average range of blood pressure in patients.

It shows systolic $94 \mathrm{~mm}$ of $\mathrm{Hg}$ and diastolic $51 \mathrm{~mm}$ of $\mathrm{Hg}$. Their blood pressure during admission does not show any significant differences. 


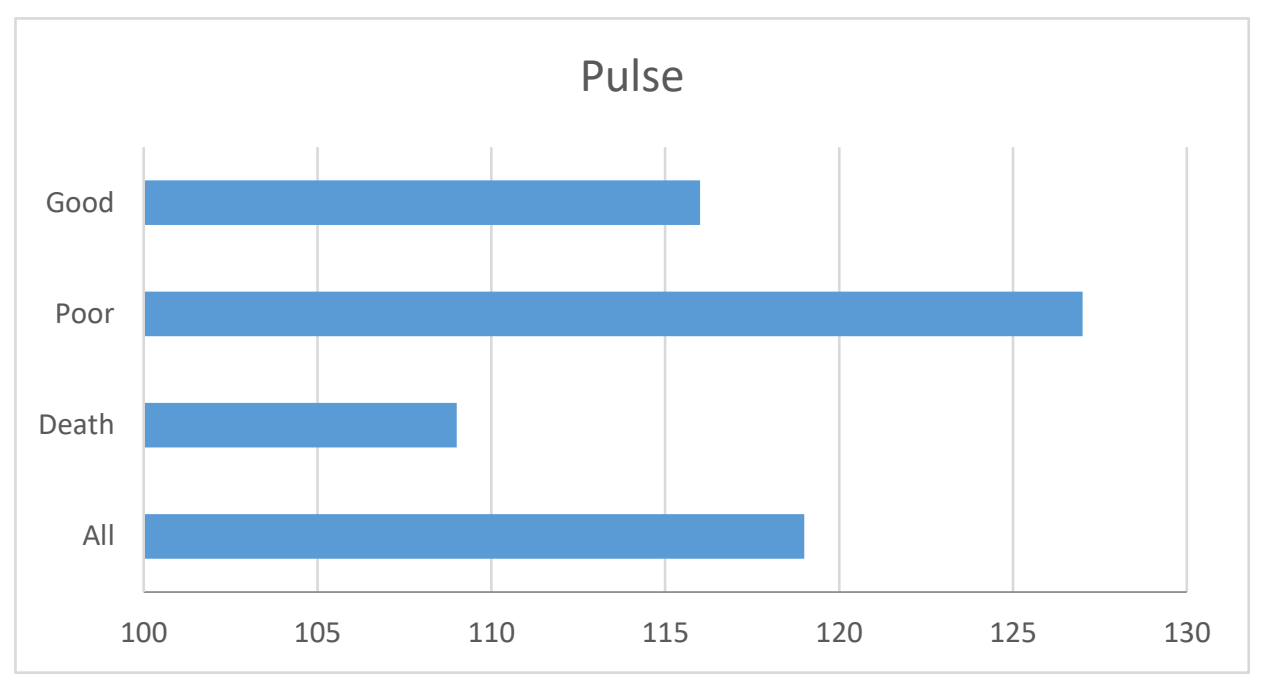

Figure 2: Pulse rate in recovered patients.

It shows an average of 116 . In poor prognosis, the patient's pulse rate is 127 , and the mortality group shows an average pulse rate of 119 .

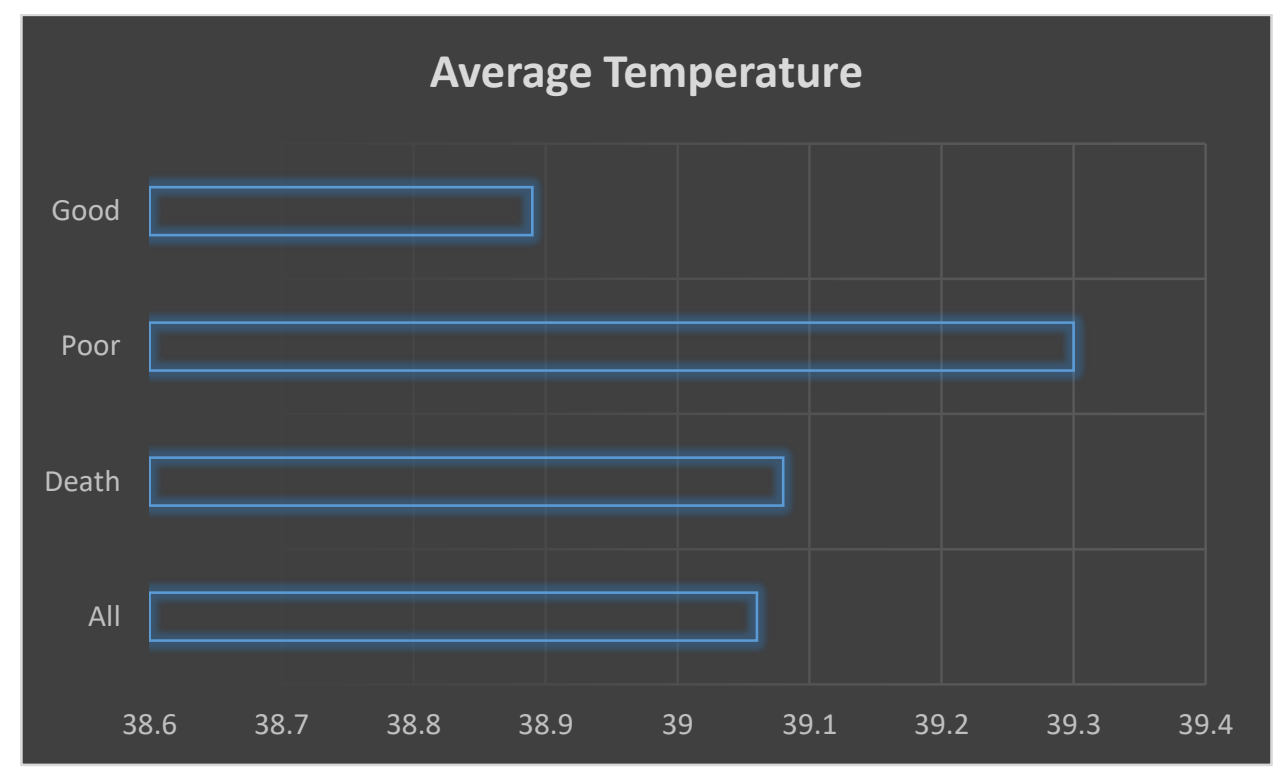

Figure 3: Mortality group.

It shows an average temperature of 39.08-degree calicoes and a poor prognosis of $39.03 \mathrm{C}$ and recovered patients with $38.89 \mathrm{C}$. 


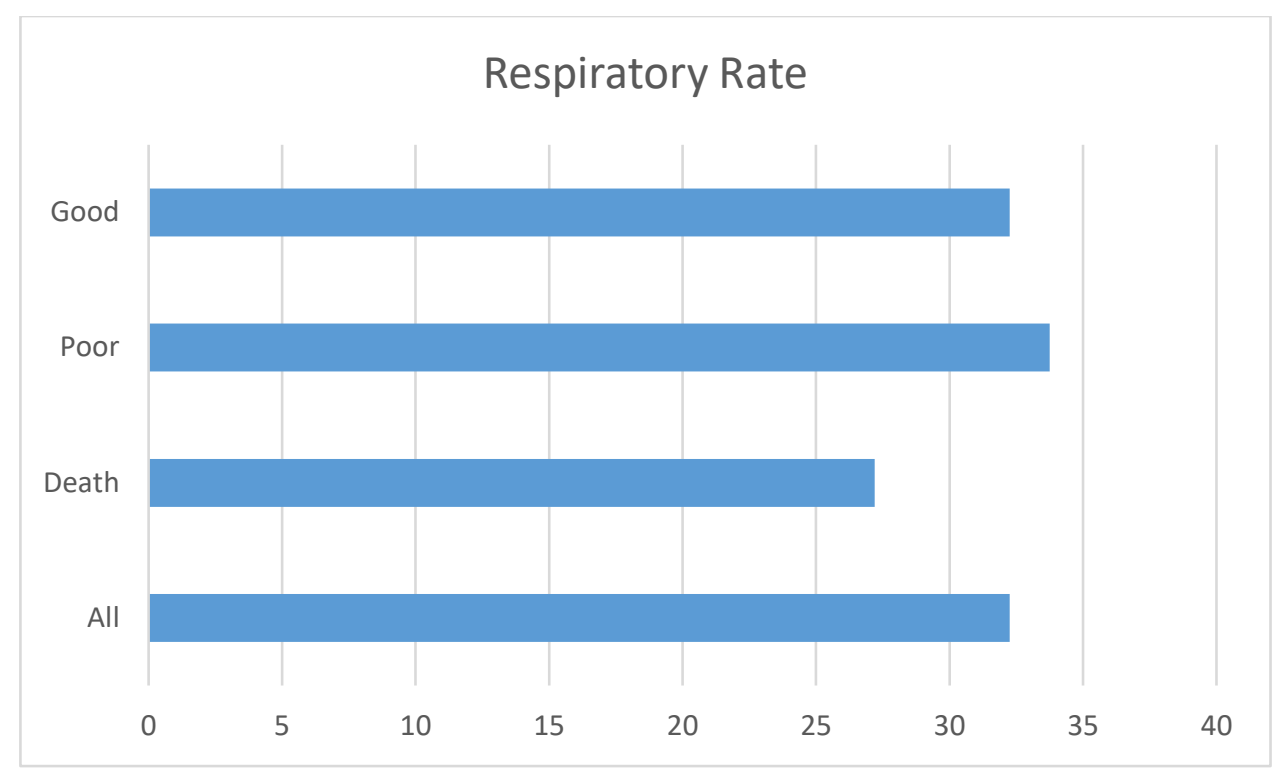

Figure 4: Death group.

It shows an average of 27.20-time respiration per minute. Poorly recovered patients showed a moderate elevation average of 33.76 times per minute with a good prognosis group 32.25 times per minute.

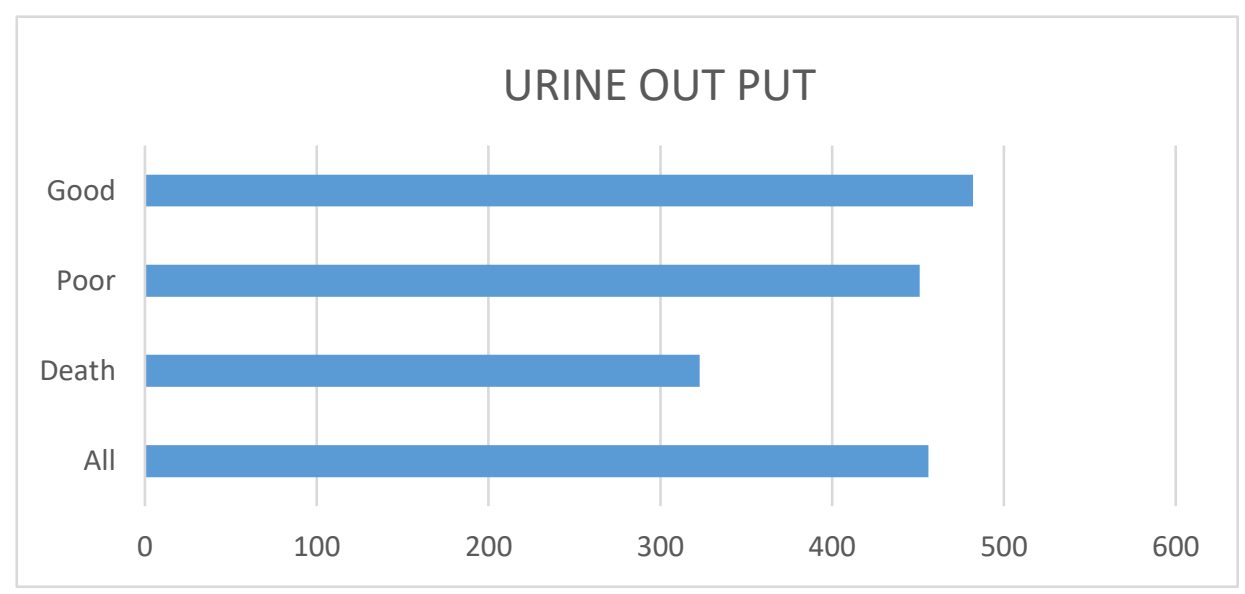

Figure 5: Urine output mortality group.

It shows an average of $323 \mathrm{ML}$. Poor prognosis group $451 \mathrm{ML}$ and the recovered group is 482 ML, which shows a greater prognosis in the recovered group of patients. 
Table 5: Mean Value of All patients LC

\begin{tabular}{lllll}
\hline Group & $\mathbf{L C}_{\mathbf{0 - 6}} \mathbf{m m o l} / \mathbf{L}$ & $\begin{array}{l}\mathbf{L C}_{\mathbf{0}-\mathbf{2}} \\
\mathbf{m m o l} / \mathbf{L}\end{array}$ & $\begin{array}{l}\mathbf{L C}_{\mathbf{0 - 2 4}} \\
\mathbf{m m o l} / \mathbf{L}\end{array}$ & $\begin{array}{l}\mathbf{L C}_{\mathbf{0}-\mathbf{4 8}} \\
\mathbf{m m o l} / \mathbf{L}\end{array}$ \\
\hline All & 3.0436 & 2.32 & 1.93 & 1.79 \\
Death & 8.08 & 4.61 & 4.25 & 5.35 \\
Poor & 3.32 & 2.55 & 2.32 & 1.91 \\
Good & 1.91 & 1.80 & 1.29 & 1.20 \\
\hline
\end{tabular}

\section{The LC development of Death Group}

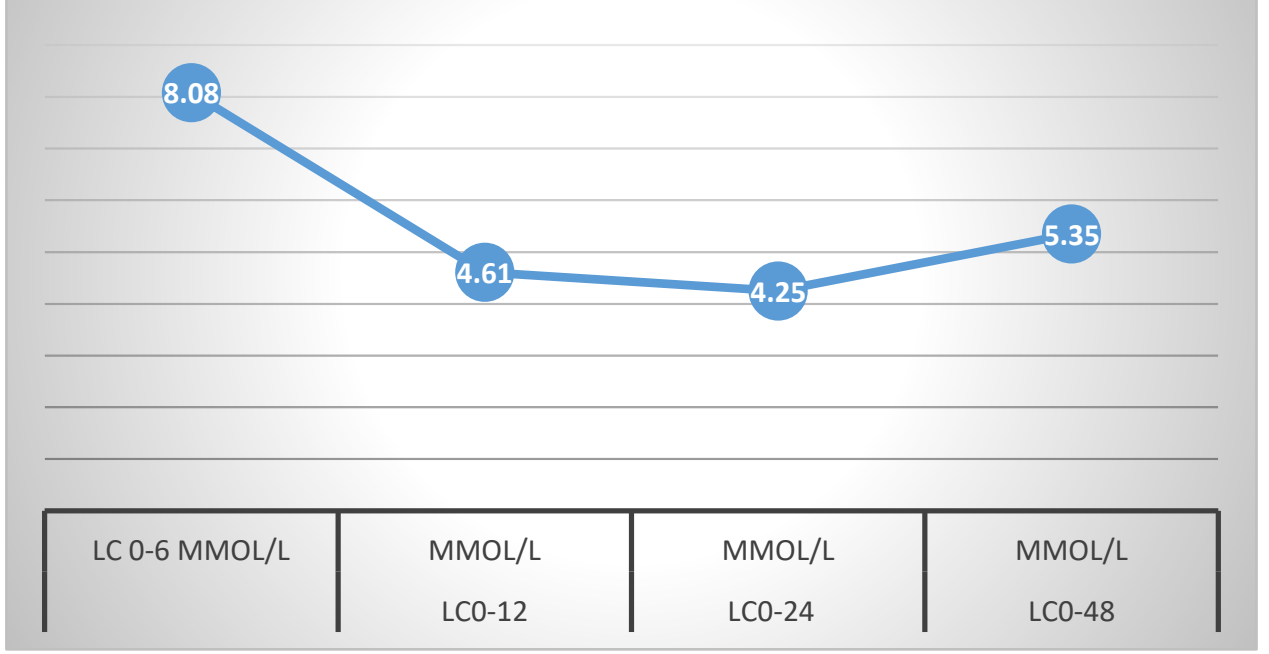

Figure 6: The LC development of the Death group.

Here it shows clearly LC in $6 \mathrm{~h}$ shows $8.08 \mathrm{MMOL} / \mathrm{L}$, which falls to $4.61 \mathrm{MMOL} / \mathrm{L}$ in $12 \mathrm{~h}$ with a slight decrease up to $4.25 \mathrm{MMOL} / \mathrm{L}$ in $24 \mathrm{~h}$ and finally in the little rise of value in $48 \mathrm{~h}$ on an average of all the patients 5.35MMOL/L. 


\section{The LC development of Poor Prognosis group}

3.32

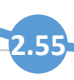

$\mathrm{MMOL} / \mathrm{L}$

LCO-12

LCO-24
$\mathrm{MMOL} / \mathrm{L}$

LCO-48

Figure 7: The LC development of Poor Prognosis group.

Here it shows clearly LC in $6 \mathrm{~h}$ shows 3.32MMOL/L which falls to $2.55 \mathrm{MMOL} / \mathrm{L}$ in $12 \mathrm{~h}$ with slightly decrease up to $2.32 \mathrm{MMOL} / \mathrm{L}$ in $24 \mathrm{~h}$ and finally ended up with the of value in $48 \mathrm{~h}$ on an average of all the patients 1.91MMOL/L.

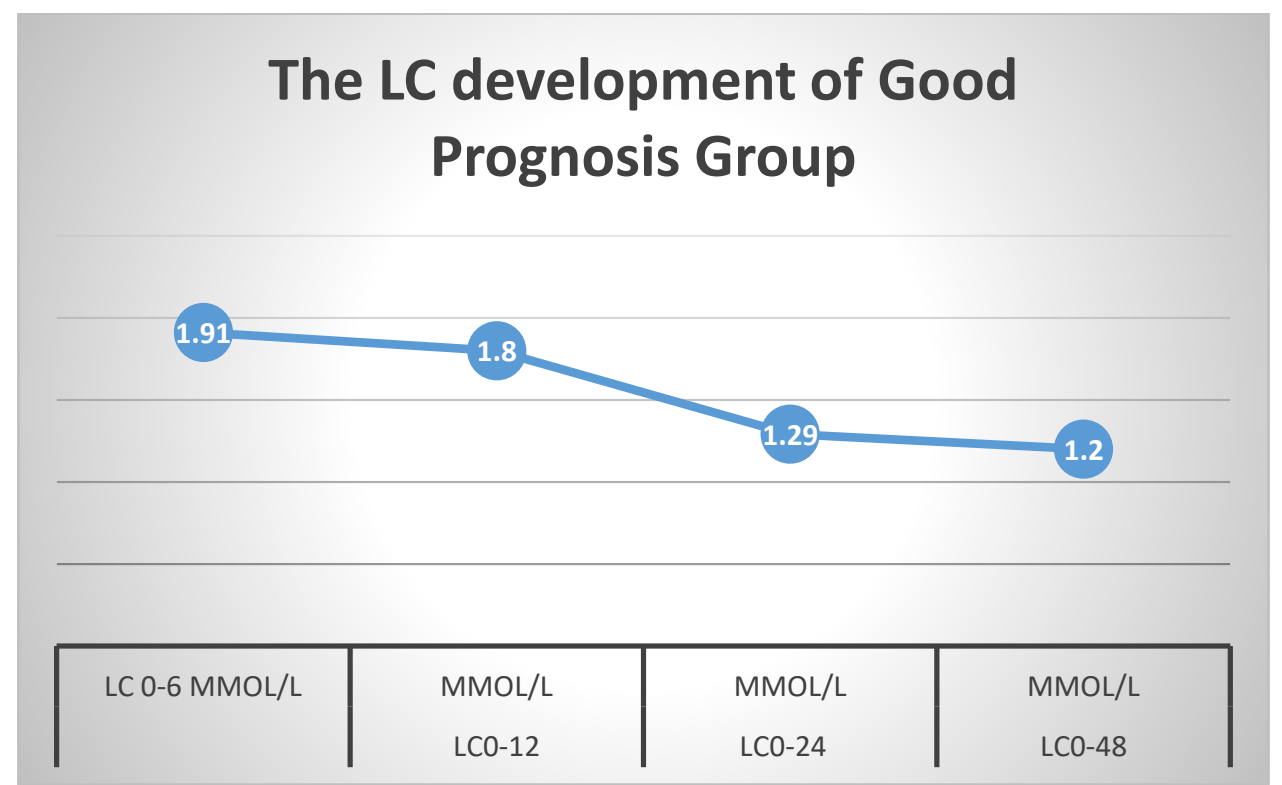

Figure 8: The LC development of a Good prognosis group.

Here it shows clearly LC in $6 \mathrm{~h}$ shows $1.91 \mathrm{MMOL} / \mathrm{L}$ which drops to $1.8 \mathrm{MMOL} / \mathrm{L}$ in $12 \mathrm{~h}$ with slightly decrease up to $1.29 \mathrm{MMOL} / \mathrm{L}$ in $24 \mathrm{~h}$ and finally ended up with the of value in 48 $\mathrm{h}$ on an average of all the recovered patients 1.2MMOL/L. from the figure we clearly can figure it out in the recovered group, we can see a clear recovery of a group of patients with a decrease of lactate clearance. 
Table 6: Patients Condition Numbers Age Limit and Duration of Stay in PICU

\begin{tabular}{llll}
\hline $\begin{array}{l}\text { Patient } \\
\text { Condition }\end{array}$ & Sex (Male/Female) & $\begin{array}{l}\text { Age Limit } \\
\text { Month-Year }\end{array}$ & $\begin{array}{l}\text { Duration of stay } \\
\text { Average }\end{array}$ \\
\hline All & Total-50(M28, F22) & $0.1-13$ & 16.86 days \\
Death & Total-5(M-5) & $0.3-11$ & 22days \\
Poor & Total-17(M-7, F10) & $0.1-13$ & 21days \\
Good & Total-28(M16, F12) & $0.1-13$ & 13.55days \\
\hline
\end{tabular}

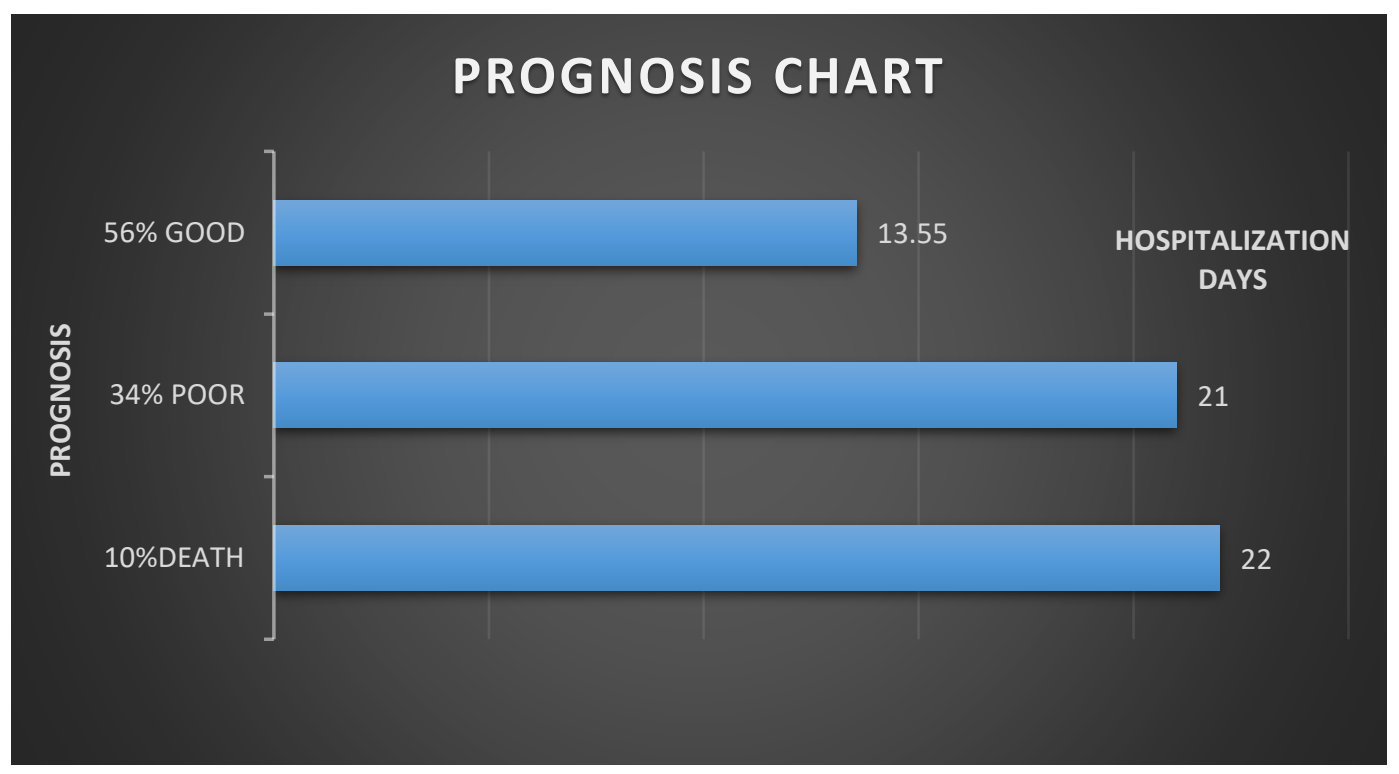

Figure 9: Hospitalization Days of Mortality group.

$34 \%$ Poor Prognosis group with 21 days of hospital admission age limit 0.1-13 years old with 7 male and 10 female patients. Good Recovery group consists of 16 male patients and 12 female patients with the age limit of 0.1-13 years old and hospitalization discharge was 13 days on an average. Mortality showed in 16 days of hospital admission and admitted patients all are male.

\section{DISCUSSION}

Many other studies have shown patients of septic shock had 50\% mortality usually. In various studies, the mortality in pediatric septic shock varied from $9.8 \%$ to $50 \%{ }^{16-21}$. We found $10 \%$ mortality and a $34 \%$ poor prognosis in our studies and a recovery rate of $56 \%$ out of the 50 patients. Mortality in our study may be due to the fact that the majority of septic shock patients admitted to PICU were fluid refractory and also refractory to one inotrope. With presently 
available bed strength, it is not possible to admit all cases with septic shock to PICU, and those who respond to fluid boluses or small doses of inotropes were managed in the wards and survived. They have not been included in our study. Therefore, patients coming to our PICU were sicker and had a higher mortality rate. In infants, the incidence of sepsis and associated mortality is higher anywhere in the world. All patients in our study were infants, so contributing to high mortality along with the poorly prognoses. In our research, we found mean LC $6 \mathrm{~h} 3.08$ $\mathrm{mmol} / \mathrm{L}$ and after $48 \mathrm{~h}$ mean is $1.79 \mathrm{mmol} / \mathrm{L}$. Significant $\mathrm{LC} 6 \mathrm{~h}$ found is $8.08 \mathrm{mmol} / \mathrm{L}$ in the death group patient were $48 \mathrm{~h} 5.35 \mathrm{mmol} / \mathrm{L}$ high. Duke et al. found that lactate allowed distinguishing survivors from non-survivors among children with sepsis at 12 and $24 \mathrm{~h}$ of admission $^{22}$. In our studies, we found significant-high lactate in the death group which is 0-6 $\mathrm{h}$ $8.08 \mathrm{mml} / \mathrm{L}, 0-12 \mathrm{~h} 4.61 \mathrm{mmol} / \mathrm{L}$ and in $24 \mathrm{~h}$ it is $4.25 \mathrm{mml} / \mathrm{L}$ and $48 \mathrm{~h}$ it is $5.35 \mathrm{mml} / \mathrm{L}$ which shows survivor rate of the patients are till $48 \mathrm{~h}$ after admission in the hospital. Hatherill et al. suggested that hyperlactatemia can indicate death on admission and if it persists after $24 \mathrm{~h}$ of treatment ${ }^{23}$. In another study, as a predictor of death, the blood lactate level at $24 \mathrm{~h}$ of PICU admission presented the best sensitivity and specificity ${ }^{24}$. These results reflect in our study as well (see tables 1-6 and figures 1-9).

Further, a lactate value of more than $45 \mathrm{mg} / \mathrm{dl}(5 \mathrm{mmol} / \mathrm{l})$ predicted death at a significant level in previous other studies by Duke et al. and Koliski et al., a lactate level of $>3 \mathrm{mmol} / \mathrm{l}$ significantly predicted mortality 22,24 . The severity of critical illness and organ dysfunction assessed using the lactate levels at presentation were similar in survivors and non-survivors' cases. However, the LCO-6 and LCO-24 were higher in non-survivors compare with nonsurvivor in our findings. Our observations suggest that LC, as defined by the percentage of lactate cleared over a period of time after disease presentation, is an independent variable associated with decreased mortality rate. Assessment of the utility of serum lactate in critically ill patients has shown that lactate levels in the emergency department and the intensive care setting have a role in risk-stratification ${ }^{25-27}$. Recovered patients showed a significant improvement after administering treatment depending on the patient's organ involvement and good decrease of lactate reports achieved, which is $1.20 \mathrm{mmol} / \mathrm{L}$, where admission reports show it was $1.91 \mathrm{mmol} / \mathrm{L}$ in LC $6 \mathrm{~h}$. But in the mortality information, we found mean LC $6 \mathrm{~h}$ $3.08 \mathrm{mmol} / \mathrm{L}$, and after $48 \mathrm{~h}$ mean it is $1.79 \mathrm{mmol} / \mathrm{L}$. Significant LC $6 \mathrm{~h}$ found, which is $8.08 \mathrm{mmol} / \mathrm{L}$ in the death group patient, was $48 \mathrm{~h} \mathrm{mmol} / \mathrm{S}$ high. Besides serial measurements, the duration and area under the curve of increased lactate levels have relationships with morbidity and mortality in different patient groups ${ }^{28-29}$. Studies have shown that during the 
most proximal stage of resuscitation, lactate levels seem to be more closely related to outcome than frequently used hemodynamic measurements, including oxygen delivery and oxygen consumption $^{29}$. We observed that mortality was high in both sets of patients, with LC0-6 of $<10 \%$ and the ones with LC0-24 of $<20 \%$. However, LC0-24 was a better predictor of mortality than LC0-6 on the comparison. Several studies in adults, in severe sepsis, pointed out the value of blood LC in the first $6 \mathrm{~h}$ of resuscitation for the prediction of day-28 survival ${ }^{30-}$ 31.

\section{CONCLUSION}

Septic shock is a common cause of PICU admission and high mortality. Lactate levels at 6, 12, and $24 \mathrm{~h} \mathrm{(>5} \mathrm{mmol/l)} \mathrm{were} \mathrm{predictors} \mathrm{of} \mathrm{death} \mathrm{in} \mathrm{septic} \mathrm{shock.} \mathrm{This} \mathrm{study} \mathrm{demonstrated} \mathrm{that}$ most patients who died had higher blood lactate levels than those who survived. There is a need for larger studies on cutoff values of lactate levels in pediatric septic shock above which mortality increases significantly. The persistence of high lactate was associated with higher mortality. This makes it useful as a prognostic marker for the risk of death. The numbers of patients were small in our study; therefore, further studies are necessary to confirm the predictive value of lactate in pediatric patients admitted to PICU. This study indicates that all three serial blood lactate levels, that is, on admission to the PICU and after 24 and $48 \mathrm{~h}$ were significantly associated with mortality in children with septic shock. Based on this study's findings, we conclude that rising or persistently high lactate levels, as shown by $<10 \%$ lactate clearance at $48 \mathrm{~h}$, is a predictor of mortality in such patients. These findings suggest an important role for serial lactate sampling rather than isolated measurement for predicting outcomes in children with septic shock.

Septic shock is one of the major causes of admission and death in intensive care units. Prompt identification of inadequate tissue perfusion and its aggressive management is essential in treating patients with septic shock, particularly with the increasing incidence and burden of managing the morbidity and mortality. Many critically ill patients, who are normotensive and have adequate urine output, which we can find in our clinical finding in the study, may remain in a state of compensated shock. Hence, relying solely on the normalization of vital signs and urine output may be inadequate. In the state of shock, anaerobic metabolism ensues, releasing lactate into the bloodstream. Elevated blood lactate levels provide an insight into the presence of impaired tissue perfusion. In recent years, lactate has been studied as a biomarker for sepsis and septic shock. Therefore, lactate clearance biologically reflects the homeostasis of the host 
and provides more meaningful data about the overall adequacy of the resuscitative processes. However, most research with serum lactate in sepsis and septic shock has been conducted in adults. Pediatric data on the association of lactate levels with mortality in sepsis and septic shock are scarce. The present study was aimed to ascertain whether lactate clearance predicts the outcome of children with septic shock admitted to the Pediatric Intensive Care Unit (PICU) and to determine the optimal cutoff value for in-hospital mortality prediction.

\section{Acknowledgements}

We much appreciate Second Affiliated Hospital of Hebei Medical University for their help.

\section{Declaration of Conflicting Interests}

The authors report no potential conflicts of interest with respect to the research, authorship, and/or publication of this article.

\section{Data and Material Availability}

Available on request to the corresponding author.

\section{Author Contributions}

DMKH: conceptualization, conducting research, statistical analysis and manuscript writing; SHE and MRK: Manuscript writing and manuscript review; XYM: manuscript review; ZH and CY: supervision, statistical analysis and critical review of the manuscript.

\section{Funding Statement:}

Not applicable.

\section{REFERENCES}

[1] Singer M, Deutschman CS, Seymour CW, et al. The Third International Consensus Definitions for sepsis and Septic Shock (Sepsis-3). JAMA 2016; 315:801-810.

[2] Dellinger RP, Levy MM, Rhodes A, et al. Surviving Sepsis Campaign Guidelines Committee including The Pediatric Subgroup. Surviving Sepsis Campaign: international guidelines for management of severe sepsis and septic shock, 2012. Intensive Care Med 2013; 39:165-228. 
[3] Rhodes A, Evans LE, Alhazzani W, et al. Surviving Sepsis Campaign: International Guidelines for Management of Sepsis and Septic Shock: 2016.Intensive Care Med 2017; 43: 304 .

[4] Kim YA, Ha EJ, Jhang WK, et al. Early blood lactate area as a prognostic marker in pediatric septic shock. Intensive Care Med 2013; 39: 1818-1823.

[5] Arnold RC, Shapiro NI, Jones AE, et al. Multicenter study of early lactate clearance as a determinant of survival in patients with presumed sepsis. Shock 2009;32: 35 .

[6] Nguyen HB, Rivers EP, Knoblich BP, et al. Early lactate clearance is associated with improved outcome in severe sepsis and septic shock. Crit Care Med 2004; 32:16371642.

[7] Bakker J, Nijsten MW and Jansen TC. Clinical use of lactate monitoring in critically ill patients. Ann Intensive Care 2013; 3: 12.

[8] Gorgis N, Asselin JM, Fontana C, et al. Evaluation of the association of early elevated lactate with outcomes in children with severe sepsis or septic shock. Pediatr Emerg Care. Epub ahead of print 9 January 2017. doi:10.1097/PEC.0000000000001021.

[9] Scott HF, Brou L, Deakyne SJ, et al. Lactate clearance and normalization and prolonged organ dysfunction in pediatric sepsis. J Pediatr 2016; 170: 149-55.e1-4.

[10] Dettmer M, Holthaus CV and Fuller BM. The impact of serial lactate monitoring on emergency department resuscitation interventions and clinical outcomes in severe sepsis and septic shock: an observational cohort study. Shock 2015; 43: 55-61.

[11] Chertoff J, Chisum M, Simmons L, et al. Prognostic utility of plasma lactate measured between 24 and $48 \mathrm{~h}$ after initiation of early goal-directed therapy in the management of sepsis, severe sepsis, and septic shock. J Intensive Care 2016; 4: 13.

[12] Jansen TC, van Bommel J, Schoonderbeek FJ, et al. Early lactate-guided therapy in intensive care unit patients: a multicenter, open-label, randomized controlled trial. Am J Respir Crit Care Med 2010; 182: 752-761.

[13] Dettmer M, Holthaus CV and Fuller BM. The impact of serial lactate monitoring on emergency department resuscitation interventions and clinical outcomes in severe sepsis and septic shock: an observational cohort study. Shock 2015; 43: 55-61.

[14] Goldstein B, Giroir B and Randolph A; International Consensus Conference on Pediatric Sepsis. International pediatric sepsis consensus conference: definitions for sepsis and organ dysfunction in pediatrics. Pediatr Crit Care Med 2005; 6: 2-8. 
[15] Brierley J, Carcillo JA, Choong K, et al. Clinical practice parameters for hemodynamic support of pediatric and neonatal septic shock: 2007 update from the American College of Critical Care Medicine. CritCare Med 2009; 37: 666-688.

[16] Llorens XS, Vargas S, Guerra F, Coronado L. Application of new sepsis definitions to evaluate the outcome of pediatric patients with severe systemic infections. Pediatr Infect Dis J 1996;14:557-61.

[17] Hatherill M, Tibby SM, Evans R, Murdoch IA. Gastric tonometry in septic shock. Arch Dis Child 1998;78:155-8.

[18] Upadhyay M, Singhi S, Murlidharan J, Kaur N, Majumdar S. Randomized evaluation of fluid resuscitation with crystalloid (saline) and colloid (polymer from degraded Gelatin in saline) in pediatric septic shock. Indian Pediatr 2005;42:223-31.

[19] Dugas MA, Proulx F, Jaeger A, Lacroix J, Lambert M. Markers of tissue hypoperfusion in Pediatric Septic Shock. Intensive care Med 2000;26:75-83.

[20] Carcillo JA, Davis AL, Zaritsky A. Role of early fluid resuscitation in pediatric septic shock. JAMA 1991;266:1242-5.

[21] Jafari HS, Mccracken GH. Sepsis and septic shock: A review for clinicians. Pediatr Infect Dis J 1992;11:739-49.

[22] Duke TD, Butt W, South M. Predictors of mortality and multiple organ failure in children with sepsis. Intensive Care Med 1997;23:684-92.

[23] Hatherill M, McIntyre AG, Wattie M, Murdoch AI. Early hyperlactatemia in critically ill children. Intensive Care Med 2000;26:314-8.

[24] Koliski A, Cat I, Giraldi DJ, Cat ML. Blood lactate concentration as prognostic marker in critically ill children. J Pediatr (Rio J) 2005;81:287-92.

[25] Zhang $\mathrm{Z}$ and $\mathrm{Xu} \mathrm{X}$. Lactate clearance is a useful biomarker for the prediction of all-cause mortality in critically ill patients: a systematic review and meta-analysis. Crit Care Med 2014; 42: 2118.

[26] Mikkelsen ME, Miltiades AN, Gaieski DF, et al. Serum lactate is associated with mortality in severe sepsis independent of organ failure and shock. Crit Care Med 2009; 37: 1670.

[27] Adeva-Andany M, Lo' pez-Oje'n M, Funcasta-Caldero' nR, et al.

Comprehensive review on lactate metabolism in human health. Mitochondrion 2014; 17: 76. 
[28] Jansen TC, van Bommel J and Bakker J. Blood lactate monitoring in critically ill patients: a systematic health technology assessment. Crit Care Med 2009; 37:28272839.

[29] Jansen TC, van Bommel J, Woodward R, et al. Association between blood lactate levels, sequential organ failure assessment subscores, and 28-day mortality during early and late intensive care unit stay: a retrospective observational study. Crit Care Med 2009; 37:2369-2374.

[30] Nguyen HB, Rivers EP, Knoblich BP, et al. Early lactate clearance is associated with improved outcome in severe sepsis and septic shock. Crit Care Med 2004; 32: 1637-1642.

[31] Tian HH, Han SS, Lv CJ, et al. The effect of early goal lactate clearance rate on the outcome of septic shock patients with severe pneumonia. Zhongguo Wei Zhong Bing Ji Jiu Yi Xue 2012; 24: 42-45. 